
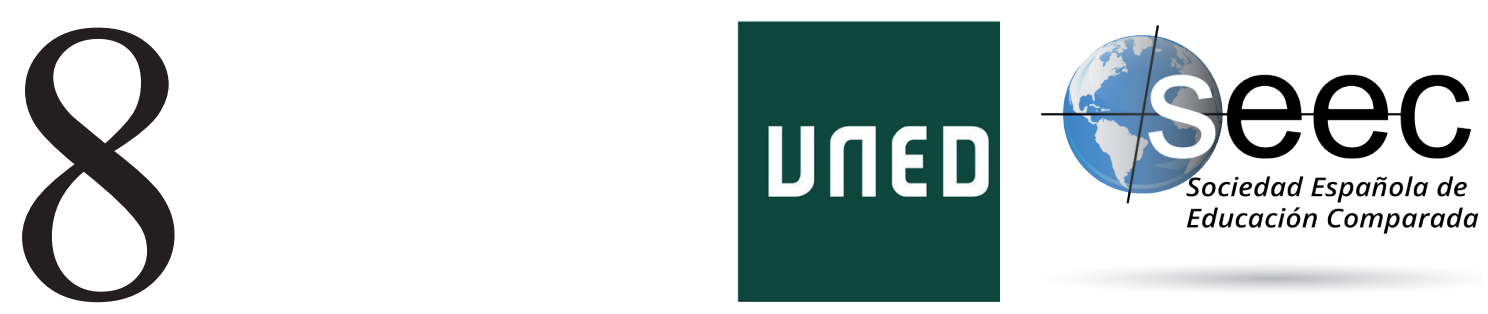

\title{
La educación terciaria tecnológica en el Mercosur. Caracterización desde una perspectiva comparada
}

Technological tertiary education in Mercosur. Characterization from a comparative perspective

\section{Irma Briasco*}

DOI: $10.5944 /$ reec.37.2021.28049

Recibido: 17 de agosto de 2020 Aceptado: 21 de octubre de 2020

\footnotetext{
*IRma Briasco: Doctora en Educación por la Universidad Nacional de Córdoba (Argentina). Desde 1995 a 2018 se desempeñó como Especialista en Educación Técnico Profesional para Iberoamérica de la Organización de Estados Iberoamericanos. Desde 2007 se desempeña como docente e investigadora en la Universidad Pedagógica Nacional, Argentina. Es miembro de la Comisión Directiva de la Sociedad Argentina de Estudios Comparados en Educación. Es secretaria editorial de RELEC. Datos de contacto: e-mail: irma. briasco@unipe.edu.ar
} 


\title{
Resumen
}

El presente artículo tiene como objetivo describir la situación, a nivel macro, de la educación terciaria tecnológica en los países de Mercosur, mediante análisis de fuentes secundarias disponibles. Recoge el desarrollo de un estudio sectorial citado en la bibliografía y en la introducción. El mismo se realizó en el marco del Núcleo de Estudios e Investigaciones en Educación Superior del MERCOSUR (NEIES) ${ }^{1}$.

Se presentan las decisiones metodológicas que guiaron el trabajo que permitieron realizar la caracterización propuesta, detallando en dos tablas la situación de la oferta de educación terciaria tecnológica.

Los hallazgos del análisis se sintetizan a modo de tendencias emergentes, para luego dejar planteado aportes para la discusión.

Se espera poder hacer un aporte en un espacio que presenta importantes lagunas, dada la falta de estudios específicos para estas ofertas.

Palabras clave: educación terciaria tecnológica; MERCOSUR; macroanálisis de la educación terciaria; perspectiva comparada.

\begin{abstract}
The objective of this paper is to describe the situation, at the macro level, of technological tertiary education in the Mercosur countries, through an analysis of available secondary sources. Collects the development of a sectorial study cited in the bibliography and in the introduction. It was carried out within the framework of the Nucleus of Studies and Research in Higher Education of MERCOSUR (NEIES) ${ }^{2}$.

The methodological decisions that guided the work that allowed to carry out the proposed characterization are presented, detailing in two tables the situation of the offer of technological tertiary education.

The findings of the analysis are synthesized as emerging trends, to then leave contributions for discussion.

It is expected to be able to make a contribution in a space that presents important gaps, given the lack of specific studies for these offers.
\end{abstract}

Keywords: technological tertiary education; MERCOSUR; macroanalysis of tertiary education; comparative perspective

1Es una de las iniciativas del Sector Educativo del MERCOSUR (SEM), que se desarrolla bajo la órbita de trabajo de la Comisión de Área de Educación Superior (CAES)

2It is one of the initiatives of the Educational Sector of MERCOSUR (SEM), which is developed under the working orbit of the Higher Education Area Commission (HEAC) 


\section{Introducción}

Este trabajo tiene como objetivo describir la situación, a nivel macro, de la educación superior tecnológica en los países participantes de Mercosur, mediante análisis de fuentes secundarias disponibles. Recoge el desarrollo de uno de los ejes del estudio sectorial La oferta de educación terciaria tecnológica. Caracterización de los modelos de gestión institucional desde la perspectiva de los estudios comparados, ${ }^{1}$ realizado en el marco del NEIES.

En las decisiones metodológicas se dejan planteadas las dificultades que esta tarea demanda, dada la carencia de sistemas de información confiables, en particular para las ofertas que son el objeto de estudio de este artículo. Para presentar el estado de situación de la educación terciaria tecnológica en los sistemas educativos se definieron una serie de dimensiones que orientaron el análisis: oferentes; duración y título; cobertura; organización y enfoque curricular; estructura y gobierno; legislación; financiamiento y sistemas de información. La información relevada a partir de fuentes secundarias, si bien no reúne las condiciones metodológicas para enmarcarse en un estudio comparado, ha permitido realizar una lectura conjunta de las dimensiones definidas y ofrecer una caracterización regional de la educación terciaria tecnológica en la actualidad.

Los hallazgos del análisis se sintetizan a modo de tendencias emergentes, para luego dejar planteado aportes para la discusión. En este último punto se indaga en las proyecciones para la construcción de la agenda de política de Mercosur para este tipo de ofertas, intentando así realizar un aporte sustantivo al proceso de integración subregional

\section{Notas metodológicas}

En este trabajo se recoge el desarrollo de uno de los ejes del estudio sectorial mencionado, referido a la descripción de la situación a nivel macro de la educación superior tecnológica en los países participantes de Mercosur, realizado mediante análisis de fuentes secundarias disponibles. Los países seleccionados corresponden al bloque subregional, Mercosur, objeto de estudio del informe sectorial tomado como insumo.

A continuación, se detallan las decisiones metodológicas que guiaron el trabajo y las acciones desarrolladas en este sentido.

Las ofertas de educación terciaria tecnológica que se recortan en este artículo son aquellas que cumplen los criterios establecido para los programas Educación terciaria de ciclo corto (CINE 5) según la Clasificación Internacional Normalizada de Educación 2011. En este sentido, la oferta de educación superior técnica/educación terciaria técnica es caracterizada a partir de los programas educativos nacionales (y sub-nacionales) y sus respectivas certificaciones. Se entiende por oferta a todo programa educativo ofrecido por una institución educativa en una unidad de servicio determinada en un año determinado ${ }^{2}$.

1 En el estudio sectorial se desarrollaron otros dos ejes referidos al modelo de gestión y las trayectorias de los equipos directivos. Fue elaborado por un equipo de investigadores de universidades de Argentina, Brasil, Chile, Paraguay y Uruguay. Fue aprobado por NEIES en junio de 2020. Dicho proceso contó con dos instancias de validación donde participaron equipos técnicos de cada uno de los países.

2 Unidad de servicio es la concreción de la oferta de un servicio educativo en una localización, sede o anexo, de una unidad o institución educativa. 
Los programas de nivel CINE 5 (educación terciaria de ciclo corto) suelen estar destinados a impartir conocimientos, habilidades y competencias profesionales. Estos programas se caracterizan por estar basados en un componente práctico, orientados a ocupaciones específicas y, por preparar a los estudiantes para el mercado laboral. Sin embargo, también pueden facilitar el ingreso a otros programas de educación terciaria. El nivel CINE 5 tiene una duración mínima de dos años y suele tener (aunque no siempre) una duración inferior a tres años.

Siguiendo a Christopher Freeman (tomado de Albornoz y Osorio, 2018) se evaluó pertinente la consulta de indicadores de nivel internacional que, según la clasificación propuesta, concierne propiamente a la comparación entre países. Esta tarea es generalmente desarrollada por organizaciones supranacionales a través de metodologías adecuadas para recopilar información, tomando como referencia los datos suministrados por los organismos encargados de la recolección en cada país. Posteriormente, se realiza un procesamiento adecuado para obtener indicadores normalizados, de modo que sean comparables entre países a nivel regional. En este nivel se pueden ubicar las diversas experiencias como la de la Red INDICES; The World Technical and Vocational Education and Training (TVET) de UNESCO-UNEVOC, o del Sistema de Información de Tendencias Educativas en América Latina (SITEAL), entre otros consultados.

Según lo propuesto por el Manual de Lima (OEI, 2017a) como primera etapa de trabajo se consideró importante avanzar en la caracterización y descripción cualitativa de los sistemas nacionales de Educación Superior, haciendo especial foco en el nivel CINE 5; y en una segunda instancia, relevar y sistematizar algunos indicadores vinculados con los estudiantes, el personal y el financiamiento de este mismo nivel. Sin embargo, dada la escasa información comparativa sobre estos últimos aspectos3, en general, en los sistemas de educación superior de los cinco países estudiados y, en particular, en el recorte CINE 5, se decidió no incorporarlos en este artículo. Si bien la clasificación CINE ofrece un marco para la descripción estadística de los sistemas nacionales de educación, es importante señalar que en la mayoría de los países de la región las estadísticas disponibles son limitadas al igual que los diagnósticos nacionales. Por ejemplo, la identificación de las instituciones oferentes y de su matrícula asociada es compleja y aún más difícil es contar con información sobre el personal, el financiamiento y los resultados de la inserción laboral de sus egresados, recortados por el nivel CINE. Según el informe de la OREALC/UNESCO (2013) en ninguno de los países estudiados se detectaron Sistemas de Información (SINFO) de la Educación y Formación Técnica y Profesional y del total de su oferta. Si bien se realizan operativos censales del sistema educativo en general, que relevan datos de la educación técnica del nivel secundario y superior técnico, no se integra con la información recogida por el sector o subsistema universitario.

Con el objetivo de realizar la descripción de los sistemas nacionales, se relevaron distintas fuentes: marcos normativos, informes ministeriales y artículos de investigaciones. Esta descripción de los sistemas nacionales, desde sus características cualitativas, buscó contextualizar la información proveniente de los indicadores elaborados por distintas organizaciones supranacionales, también relevados para este estudio. Esta descripción cualitativa permite dar cuenta en cada país de:

3 En relación con el Personal solo se encontró información parcial para Argentina (personal del sector de gestión público universitario), Brasil y Chile. 
-La organización del sistema de educación superior: marco normativo, organización interna, subsistemas, jurisdicción o nivel del que depende, organismos de regulación, planificación y coordinación y organismo de evaluación.

-Las instituciones del sistema de educación superior: tipo de instituciones, número de instituciones según la clasificación establecida, número de instituciones por subsistema, número de instituciones por sector de gestión.

-Las propuestas de formación: normativa, cantidad de programas, cantidad de programas por subsistema, cantidad de ofertas por subsistema.

Para conocer el estado de situación de la educación terciaria tecnológica en los sistemas educativos se definieron una serie de dimensiones que orientaron el análisis: oferentes; duración y título; cobertura; organización y enfoque curricular; estructura y gobierno; legislación; financiamiento y sistemas de información. La información relevada a partir de fuentes secundarias, si bien no reúne las condiciones metodológicas para enmarcarse en un estudio comparado, ha permitido realizar una lectura conjunta de las dimensiones definidas y ofrecer una caracterización regional de la educación terciaria tecnológica en la actualidad.

\section{La Educación Terciaria Tecnológica en el MERCOSUR}

\subsection{Puntos de partida}

Las ofertas impartidas en los institutos tecnológicos de los países seleccionados, se encuentran enmarcadas en las respectivos lineamientos nacionales referidos a «enseñanza y formación técnica y profesional» (en adelante EFTP). En este artículo se considera la definición acordada en UNESCO:

«comprende la enseñanza, la formación y la adquisición de destrezas relativas a una gran variedad de sectores ocupacionales, actividades de producción, servicios y medios de subsistencia. La EFTP, como parte del aprendizaje a lo largo de toda la vida, puede impartirse en los niveles secundario, postsecundario y superior, e incluye el aprendizaje en el trabajo y la formación permanente y el desarrollo profesional que pueden conducir a la obtención de certificaciones. La EFTP incluye asimismo una gran variedad de posibilidades de adquisición de destrezas en función de los contextos nacionales y locales. Aprender a aprender, el desarrollo de aptitudes en materia de lectoescritura y de cálculo y las aptitudes transversales y para la ciudadanía forma parte integral de la EFTP» (UNESCO, 2015a).

Los países de América Latina y el Caribe presentan diferentes denominaciones y alcances para la EFTP. El punto de partida es, entonces, el reconocimiento de la heterogeneidad y complejidad que la EFTP y sus sistemas de educación tienen en los cinco países que forman parte de este estudio. Heterogeneidad que se expresa tanto en relación con la estructura, como con la institucionalidad y el gobierno del sistema. 
Asimismo, el mencionado estudio de UNESCO señala que los sistemas nacionales de educación superior han experimentado una fuerte expansión durante las últimas décadas producto del aumento continuo de la participación de la población en este nivel educativo. En respuesta a una demanda cada vez más masiva y diversa, los sistemas amplían y diversifican también su oferta mediante la conformación de sistemas crecientemente diferenciados en su base institucional. Por lo tanto, la heterogeneidad propia de la EFTP suma complejidad al entramarse con el dinamismo y diversificación que asume el nivel superior. La diversificación y expansión de los sistemas de educación superior puede interpretarse como respuesta ante la creciente masificación del nivel, pero también como parte del dinamismo de la EFTP. Este fenómeno se manifiesta en: a) la creación de instituciones de educación terciaria con programas de ciclo corto que, por sus objetivos y su mayor vinculación con la producción y los intereses de los territorios, resultan pertinentes y distintas a las universidades tradicionales; y b) la reducción del tiempo necesario para el egreso de los estudiantes de las universidades mediante la creación de los llamados niveles de titulación.

Por su parte, el informe «Estrategia para la enseñanza y formación técnica y profesional (EFTP) (2016-2021)» destaca que uno de los factores con influencia en la expansión y diversificación tanto de la educación superior en general, como de la superior técnica en particular, es la extensión de la duración del período de educación obligatoria establecida en las leyes de educación o normas nacionales (UNESCO, 2016). En esta misma línea, Jacinto (2013, citado en Briasco, 2018) analiza esta expansión y diversificación y sostiene que la misma sucede principalmente en países con una alta demanda de educación superior, que buscan aumentar la cobertura del nivel diversificando el modelo universitario tradicional y la formación de grado de larga duración. En un estudio posterior de carácter comparativo (tomando casos de Brasil, Colombia y México), esta misma autora se pregunta: «¿la «educación técnica terciaria» ha contribuido a aumentar las oportunidades económicas y sociales de los jóvenes en América Latina?» (Jacinto y Fanelli, 2014; citado en Briasco, 2018). El análisis muestra que la educación técnica de nivel terciario (ETT) ha contribuido efectivamente a generar más oportunidades en la medida en que constituye una instancia de formación alternativa para las nuevas generaciones. Se señala además un tema central para la orientación de intereses: el de la representación social de esta oferta.

«La ETT es más accesible, de menor duración, tiene una orientación vocacional y es en general más barata que la formación universitaria. No obstante, los estudios de caso también revelan que, si bien un título de educación técnica terciaria es un activo para los jóvenes que buscan empleo, no se le otorga el mismo valor que a un título universitario tradicional» (Jacinto, Fanelli, op. cit.).

En el mismo trabajo se señala además que el diploma tiene una circulación de mercado diferenciada por sector, siendo más valorado en el ámbito de los servicios, en algunas ocupaciones reguladas y, en menor medida, en el sector industrial. Se destaca asimismo un hallazgo relevante para el presente estudio, referido a las trayectorias educativas de los graduados: «el proceso de articulación e integración entre la educación no universitaria y universitaria continúa siendo incipiente. Las transiciones exitosas entre la educación técnica terciaria y el sistema universitario parecen ser escasas» (Jacinto, Fanelli, op. cit.). 
Estos aportes desde estudios comparativos, en particular en el marco de un proceso de integración subregional como es el de Mercosur, suponen primeros esfuerzos en la línea de construcción de políticas educativas supranacionales, cuyo objeto se define como «un área emergente del saber pedagógico, en íntima conexión con la Política Educativa, la Educación comparada y la Educación Internacional; tratando de acotar su objeto de estudio y apuntar sus áreas de interés propio» (Valle, 2013)

\subsection{Caracterización de la Educación Tecnológica en cada país de MERCOSUR}

\subsubsection{Argentina}

En Argentina es el Instituto Nacional de Educación Tecnológica, dependiente del Ministerio de Educación Nacional, el organismo rector para la construcción de las políticas educativas Educación Técnico Profesional de Nivel Superior, que permite iniciar y/o continuar itinerarios profesionalizantes, a través de una formación en áreas ocupacionales específicas cuya complejidad requiere el dominio y la manifestación de conocimientos, habilidades, destrezas, valores y actitudes profesionales que solo son posibles de desarrollar a través de procesos de formación prolongados y sistemáticos.

El ámbito y el Nivel de Educación Superior se caracterizan por los rasgos peculiares de sus instituciones. Ellas deben desarrollar funciones relativas a la enseñanza, la investigación, el desarrollo, la vinculación tecnológica y la innovación. Se garantiza a los estudiantes una base de conocimientos, habilidades y una trayectoria profesional, orientadas a lograr una mejor inserción y desarrollo en un campo profesional determinado del mundo del trabajo. Asimismo, posibilita la continuidad del aprendizaje durante toda su vida activa. La formación en este ámbito y nivel debe complementarse con otras alternativas educativas de perfeccionamiento para permitir ulteriores niveles de evolución, especificación, reorientación, y, eventualmente, de reconversión de la profesión inicial. En este sentido, es necesario favorecer una articulación dentro de la modalidad Técnico Profesional a través del desarrollo de diferentes trayectorias posibles: La Educación Técnico Profesional inicial de nivel Secundario; la Formación Profesional Continua; la Educación Superior, que ofrece el ámbito universitario y las jurisdicciones educativas provinciales y la Formación Profesional Inicial.

La consolidación de la identidad de la educación terciaria tecnológica es uno de los ejes de discusión más relevantes que atraviesan a esta modalidad en la Argentina, como consecuencia de un proceso de transformación que ha afectado particularmente a estas ofertas formativas y frente al cual no hubo una respuesta debidamente planificada.

Se destaca el incremento sostenido de la población que accede y egresa del nivel secundario -establecido como obligatorio hace más de una década por la Ley de Educación Nacional $\mathrm{N}^{\circ}$ 26.206/o6- ha generado una mayor demanda sobre la educación post-secundaria en general y sobre las instituciones de educación terciaria tecnológica en particular. Esto derivó en un fuerte crecimiento de la matrícula en estas ofertas y en una diversificación de la demanda en parte por el ingreso de sectores de la población que tradicionalmente no accedían a este nivel educativo. Por otro lado, se advierte un incremento de las ofertas formativas de nivel superior técnico que han buscado atender a esta nueva demanda a partir de criterios y modos diferentes, generando un panorama de mayor heterogeneidad.

Un rasgo distintivo de este proceso de expansión de la oferta ha sido el fuerte dinamismo de las instituciones formativas del sector privado y la tendencia a generar ofertas 
mayormente vinculadas con el sector servicios. Es posible que esta tendencia se explique por diferentes motivos, entre los cuales se encuentran los procesos de tercerización de la economía que se dieron en Argentina y en muchos países de América Latina en las últimas décadas, así como también la creciente relevancia que ha adquirido la administración como una actividad transversal a los diferentes tipos de organizaciones, junto con los avances tecnológicos que han incorporado el uso de sistemas informáticos en numerosas actividades como un elemento central de sus procesos. No obstante, es posible considerar también la incidencia de factores propios del sistema formador que favorecieron este tipo de crecimiento, como por ejemplo la mayor simplicidad que implica generar este tipo de ofertas formativas en cuanto a recursos disponibles.

Un tema no menor, es la necesidad de consolidar la identidad de la educación terciaria tecnológica mediante un trabajo de diferenciación y a la vez de articulación con otras ofertas que componen el espectro amplio de la EFTP en Argentina: las tecnicaturas de nivel secundario, la formación continua y las universidades. Es prioridad consensuar una definición clara del perfil profesional propio de la formación técnica de nivel superior, y al mismo tiempo diseñar estrategias institucionales que permitan articular ofertas con miras a ampliar las posibilidades de acceso a los distintos niveles de la educación técnico profesional, generando diversidad de alternativas y garantizando oportunidades de progresión en las trayectorias calificantes.

\subsubsection{Brasil}

La Educación Profesional Tecnológica (EPT) en Brasil está conformada, de acuerdo con el párrafo 2 del artículo 39 de la Ley de Directrices y Bases de la Educación Brasileña (LDB), la Ley 9.394 / 1996, por una amplia gama de actividades que cubren cursos de capacitación inicial y continua o formación profesional; educación técnica en la escuela secundaria y; educación tecnológica profesional de pregrado y posgrado. A pesar de esta complejidad, «la EPT brasileña reproduce la estructura de una sociedad caracterizada por grandes antagonismos» (Freyre, 2014) y esta «marcada por la degradación del trabajo manual» (Cunha, 2005). El legado de la esclavitud, la educación que se forma para el trabajo, para la producción de vida material, todavía está culturalmente asociada con la pobreza y la servidumbre.

Dentro del marco del Ministerio de Educación, la Secretaría de Educación Profesional y Tecnológica (SETEC / MEC) es responsable de planificar y desarrollar la Red Federal de Educación Profesional, Científica y Tecnológica, lo que incluye garantizar una disponibilidad presupuestaria y financiera adecuada. Creada en 2008 por la Ley $\mathrm{N}^{\circ} 11.892$, la Red Federal ha sido un hito en la expansión, internalización y diversificación de la educación profesional y tecnológica en el país. La misma fue aumentó el número de plazas para EPT, ofreciendo desde educación básica hasta posgrados, incluso formación inicial y continua para docentes, configurando así un cambio de paradigma en Brasil.

No obstante estos avances, mediante la Ley $\mathrm{N}^{\circ} 13.415$ en 2017 se implementa una reforma del nivel secundario que afecta también a la educación profesional y marcó el retorno del viejo y recurrente modelo de educación dual.

«Reaparecen desafíos que parecían superados. Existen semejanzas con el modelo de la década de 1990, cuando se hicieron cambios profundos mediante instrumentos legales abreviados, sin más discusiones con la sociedad, buscando la yuxtaposición con el mercado que exigía una capacitación técnica más rápida y más corta y se centró en la versatilidad» (Afonso y Gonzalez 2018). 
La llamada «nueva escuela secundaria» propone la flexibilidad de la matriz curricular basada en la definición de itinerarios formativos y tendrá como referencia la Base Curricular Nacional Común (BNCC). El nuevo modelo se refiere a una formación profesional fragmentada y desarrollada en la etapa final de la escuela secundaria, es decir, como una de las posibilidades de un itinerario formativo. A pesar de este contexto adverso, actualmente, de acuerdo con las Directrices Curriculares Nacionales para la Educación Técnica Profesional de Nivel Medio (Resolución CNE N ${ }^{\circ} 6$ de 2012), se desarrolla la Educación Técnica de Nivel Medio en forma articulada y posterior a la escuela secundaria. La formación posterior, se desarrolla en cursos destinados exclusivamente a aquellos que ya han terminado la escuela secundaria. Este formato tiene definición de capacitación técnica y no debe confundirse con la educación superior o universitaria en Brasil.

Los Institutos Federales (IF) son instituciones de educación profesional y tecnológica, reconocidas por la sociedad, con un papel notable en el desarrollo económico, social y cultural a nivel nacional, alentadas por la expansión acelerada en los últimos 10 años. El objetivo es capacitar a los ciudadanos para trabajar en los diversos sectores de la economía con énfasis en el desarrollo local, regional y nacional. Su gran diferencial se apoya en el tipo de capacitación brindada, que debe estar en línea con los principios de la educación profesional y sus bases conceptuales. En este sentido, busca superar la formación dual. Los IF son responsables de asumir la cuestión del trabajo como un principio educativo, contribuyendo a la emancipación y la autonomía de los sujetos, en la perspectiva de contribuir a la reducción de las desigualdades sociales.

Como instancia clave para garantizar la continuidad de la red federal:

a) fortalecer la educación y capacitación permanente de los funcionarios públicos, maestros y técnicos, con énfasis en la singularidad de los objetivos, misión, propósitos y características de la Red Federal Brasileña de Educación Profesional y Tecnológica, especialmente en lo que respecta a su historia, consolidación y cambios experimentados a lo largo de los años; institucionalización de programas de capacitación permanente para funcionarios que brinden subsidios para la renovación y actualización de debates recientes sobre la educación profesional tecnológica brasileña y también;

b) garantizar el acceso, la inclusión, la permanencia y el éxito de los estudiantes: es necesario asegurar un presupuesto para la continuidad de las acciones de asistencia estudiantil, garantizando lo que establece el Programa Plan Nacional de Asistencia al Estudiante (PNAES) ${ }^{4}$ instituido en 2010, que tiene varios ejes de atención a los estudiantes.

Aun así, se destaca la necesidad de una discusión permanente y la construcción de estrategias para ofrecer cursos, considerando las demandas sociales (y no solo las del mercado), la región en la que opera y la capacidad de absorción de los graduados a nivel local y regional y nacional.

\subsubsection{Chile}

En Chile la oferta se encuentra inserta en el Ministerio de Educación, el cual cuenta con una subsecretaría de educación superior. En la página oficial de Mineduc se define ${ }^{5}$ :

4 http://legislacao.planalto.gov.br/legisla/legislacao.nsf/Viw_Identificacao/DEC\%20

7.234-2010?OpenDocument. Visitado abril 2020

5 https://educacionsuperior.mineduc.cl/educacion-superior-tp/. Visitado abril 2020 
«la Formación Técnico-Profesional como un sistema único e integrado que vincule la Educación Media y la Educación Superior Técnico-Profesional con la capacitación. De esta manera, serán posibles trayectorias continuas entre los distintos momentos formativos y laborales que den respuestas a las necesidades sociales y de los sectores productivos, para que nuestros técnicos puedan insertarse en el mundo laboral con éxito. Una mejor calidad de la Formación Técnico-Profesional los beneficia directamente, potenciando sus trayectorias educativas.» (Mineduc, 2020)

Se verifica un redimensionamiento de la oferta, identificando puntos clave tales como: la Formación Técnico-Profesional como una opción realmente competitiva y con altos estándares de calidad; articulación entre la Educación Media TP, la Educación Superior TP y el sector productivo; aumento de instituciones y becas; Conformación del Consejo Nacional Técnico-Profesional; implementar Centros de Formación Técnica (CFT) estatales con calidad, pertinencia con la región y en articulación con los liceos técnico-profesionales.

Uno de los ejes del debate actual sobre la EFTP es justamente el vínculo que las instituciones formativas entablan con el entorno socio-productivo. Los planteos sobre este punto se orientan a superar la relación basada en la necesidad de realizar prácticas profesionalizantes para avanzar en un modelo de vinculación con el medio que propicie el desarrollo y la competitividad de las regiones en las cuales las instituciones se insertan y, en última instancia, el del país en su conjunto. Para ello, se postula el principio de pertinencia de la formación ofrecida, considerando que la educación terciaria tecnológica debe establecer una sinergia positiva con su entorno local y regional no solo para fomentar la colocación laboral de sus estudiantes y egresados sino también para anticipar y responder a las demandas de innovación y cambio que experimenta el ámbito productivo en su proceso de desarrollo. En vistas de este objetivo, las alianzas estratégicas entre el sector público y privado, así como entre empresas y universidades, se ponderan como instrumentos para integrar de manera efectiva y sistemática la formación con el entorno socio-productivo.

\subsubsection{Paraguay}

La Educación y Formación Técnica y Profesional se reconoce como una modalidad del sistema educativo paraguayo que brinda posibilidades de cualificación laboral en diversos niveles y especialidades de los sectores industrial, agropecuario y de servicios. Las primeras experiencias formales en esta modalidad se registraron ya en los inicios del siglo XX, sin embargo, no fue sino a partir de la década de los 80 que la Educación Técnica y Profesional cobró un impulso significativo como estrategia para la generación de recursos humanos calificados para el sector productivo del país.

Se cuenta con una oferta de formación profesional para jóvenes y adultos gestionada por la Dirección General de Educación Permanente para personas jóvenes y adultas del MEC. Dicha oferta comprende por una parte un grupo de cursos no formales de corta duración dirigidos a jóvenes y adultos mayores de 15 años que han concluido el $3^{\circ}$ Grado de la Educación Escolar Básica, denominados de Formación Profesional Inicial (FPI). De igual manera, otro grupo de cursos están dirigidos a una población joven y adulta que ha concluido el $6^{\circ}$ Grado de la Educación Escolar Básica y se denominan de Formación Profesional de Nivel 1 (FP1). Un tercer grupo de cursos se dirige a jóvenes y adultos que 
han concluido el $9^{\circ}$ Grado de la Educación Escolar Básica y se denominan de Formación Profesional de Nivel 2 (FP2). Tales cursos poseen estructuras modulares flexibles organizadas en familias profesionales que abarcan los siguientes campos ocupacionales: artesanía, diseño, corte y confección, belleza integral, idiomas, hospitalidad, administración y oficina, informática y construcciones.

En el nivel medio, gestionado por la Dirección General de Tercer Ciclo de la Educación Escolar Básica y la Educación Media del MEC, se imparten programas de bachillerato técnico ofrecidos en distintas especialidades de los sectores industrial, agropecuario y de servicios. Estos bachilleratos tienen como finalidad preparar a los estudiantes para acceder a la educación superior y desarrollar al mismo tiempo competencias profesionales para su inserción al mundo del trabajo. Los programas formativos tienen 3 años de duración, con diseños curriculares disciplinares, y están dirigidos a jóvenes que han concluido los 9 años de la Educación Escolar Básica. Actualmente, se encuentran habilitadas 28 especialidades del bachillerato técnico, lo cual ofrece a los jóvenes una amplia variedad de oportunidades para potenciar sus aptitudes y vocaciones. Otro grupo de ofertas de antigua data en el nivel medio lo constituyen los denominados programas de formación profesional en centros vocacionales. Actualmente, estos programas se encuentran en proceso de análisis para ser reconvertidos a la modalidad de formación profesional media para la formación en áreas relacionadas con la producción de bienes y servicios.

Las carreras de tecnicatura superior se imparten en universidades, institutos superiores o institutos técnicos superiores -la mayor parte se concentran en el sector privadocon diseños curriculares de entre 1600 y 2000 horas pedagógicas, distribuidas en un período no inferior a 2 años y no mayor a 3 años; con una carga adicional de 500 horas reloj mínima de pasantía profesional. Los cursos o cursos-talleres de pregrado en el nivel superior se desarrollar en un tema o disciplina determinada en el área de competencia de la institución, siendo de corta duración y con un perfil de entrada post-bachillerato y/o post-titulación de Técnico/a Superior para complementar su formación. Estos cursos son autorizados para su implementación por un lapso de un año y tienen una carga horaria mínima de 40 y máxima de 300 horas pedagógicas.

La promulgación de la reciente ley $\mathrm{N}^{\circ} 5749 / 2017$ que establece la carta orgánica del Ministerio de Educación y Ciencias ha generado un nuevo ordenamiento administrativo de la EFTP en Paraguay, incorporando una instancia consultiva denominada «Consejo Nacional de Educación y Trabajo» (CNET). Dicho organismo tiene por objeto, entre otras cuestiones, proponer y aprobar un plan estratégico de educación y trabajo que contenga acciones de implementación de la educación técnica y la capacitación laboral, propiciando la coordinación con los diversos sectores involucrados en la ejecución de las políticas públicas en materia de educación y trabajo; garantizar la implementación del Sistema Nacional de Cualificaciones Profesionales; cooperar en la implementación del Catálogo Nacional de Perfiles Profesionales.

\subsubsection{Uruguay}

El caso de Uruguay es peculiar dada la tradición que tiene este país en lo que refiere a la política, la administración y gestión de la educación pública en todas sus modalidades. La Administración Nacional de Educación Pública (ANEP) es el organismo estatal responsable de la planificación, gestión y administración del sistema educativo público en sus niveles de educación inicial, primaria, media, técnica y formación docente en todo el territorio uruguayo. 
La aprobación de la Ley General de Educación en 2008, $\mathrm{N}^{\mathrm{0}} 18.437$ mantiene a la ANEP y crea el Sistema Nacional de Educación (SNE) y el Sistema Nacional de Educación Pública (SNEP). En el art. 62 del Capítulo VI del título III referido al Sistema Nacional de Educación Pública, expresa que «El Consejo de Educación Técnico-Profesional (UTU) tendrá a su cargo la formación profesional (básica y superior), la educación media superior técnica tecnológica (bachilleratos tecnológicos), la educación media superior orientada al ámbito laboral y la educación terciaria técnica (tecnicaturas)». Es de destacar que en esta Ley se estableció la creación de una nueva institución para la Educación Superior:

«Créase el Instituto Terciario Superior (ITS) en el ámbito del Sistema Nacional de Educación Pública. Estará constituido por una red de instituciones ubicadas en diferentes lugares del país. Desarrollará actividades de educación terciaria, integrando enseñanza, investigación y extensión. Formará técnicos en diversas áreas de la producción y los servicios. Los conocimientos y créditos adquiridos serán reconocidos o revalidados por las demás instituciones terciarias o universitarias, de forma de facilitar la continuidad educativa de sus estudiantes y egresados» (art. 87).

El CEPT-UTU promueve una oferta educativa amplia y heterogénea, diseñada para un público diverso a través de más de doscientos cursos en las áreas agraria, deportiva, de servicios, de producción y artística. Acceden a estos cursos los egresados de primaria, de educación media básica pública o privada, así como egresados de educación media superior que aspiran los cursos técnicos o tecnológicos terciarios. También se ofrecen cursos de formación profesional básica para adolescentes, jóvenes y adultos que, tras abandonar sus estudios, desean continuar con su educación, y a los trabajadores que pueden acreditar sus saberes o asistir a cursos o a capacitaciones profesionales.

En diciembre de 2012, por Ley $\mathrm{N}^{\circ} 19043$ se crea la Universidad Tecnológica del Uruguay (UTEC), ente autónomo que se autodefine como una institución «de educación pública, de perfil tecnológico, que nace comprometida con la ampliación de la oferta terciaria universitaria en el interior de Uruguay, fomentando el vínculo con el medio productivo y la promoción del desarrollo social y cultural del país» ${ }^{6}$. La UETC tiene tres Institutos Tecnológicos Regionales (ITR) que ofrecen carreras tecnológicas diferentes en cada sede. De esta manera, en la actualidad hay dos instituciones públicas encargadas específicamente de la EFTP: CETP-UTU y UTEC, además de la Universidad de la República que también oferta algunos cursos técnicos terciarios, en algunos casos en convenio con CETP-UTU y UTEC.

\subsection{Sistematización de los acuerdos alcanzados en Mercosur Educativo}

El Sector Educativo del Mercosur (SEM) es un espacio de coordinación de las políticas educativas que reúne a los Estados Parte y los Estados Asociados. El mismo tuvo su origen en diciembre de 1991 cuando, a través de la Decisión 07/91, se creó la Reunión de Ministros de Educación del Mercosur. La misión del SEM es conformar un espacio educativo común, que permita estimular la movilidad y el intercambio a través de la de la negociación y concertación de políticas, la elaboración de programas y proyectos conjuntos que promuevan un desarrollo articulado de la educación en los países de la región.

6 Fuente: página oficial de la UTEC: https://utec.edu.uy/institucional/ 
A los fines del presente artículo, se destaca que, los programas y proyectos inscriptos en el área de Educación Superior del Mercosur Educativo no contemplan específicamente la educación terciaria tecnológica, en tanto se orientan al sistema de formación universitaria.

Basándose en la experiencia de trabajo acumulada por la CAET en la homologación de titulaciones técnicas para el Nivel Medio, actualmente la mencionada subcomisión se encuentra efectuando una compilación de las ofertas de los países miembros con el objetivo de avanzar en la generación de tablas de equivalencia para el reconocimiento de títulos técnicos de Nivel Superior en distintas especialidades.

\subsection{Oferta de educación terciaria tecnológica en los países de Mercosur. Tablas comparativas.}

La siguiente tabla presenta las principales particularidades de la provisión de educación terciaria tecnológica dando cuenta de la heterogeneidad institucional y de certificaciones existente.

Tabla 1.

Provisión de educación terciaria tecnológica (CINE 5), certificación que otorga y años de duración

\begin{tabular}{|c|c|c|c|}
\hline País & Instituciones oferentes & Certificación que otorgan & Duración \\
\hline \multirow[t]{3}{*}{ Argentina } & $\begin{array}{l}\text { Institutos superiores Institutos } \\
\text { tecnológicos }\end{array}$ & Técnico Superior & $2-3$ \\
\hline & 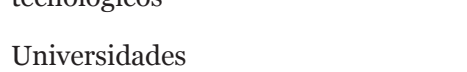 & $\begin{array}{l}\text { Técnico universitario, analista, títulos } \\
\text { intermedios }\end{array}$ & $2-3$ \\
\hline & Institutos universitarios & & \\
\hline \multirow[t]{6}{*}{ Brasil } & Red Federal de Educación & Técnico de nivel medio & $1-2$ \\
\hline & Redes Estaduales de Educación & & \\
\hline & Redes Municipales de Educación & & \\
\hline & Servicios Nacionales de Aprendizaje & & \\
\hline & Escuelas Técnicas Privadas & & \\
\hline & $\begin{array}{l}\text { Instituciones Privadas de Educación } \\
\text { Superior }\end{array}$ & & \\
\hline \multirow[t]{4}{*}{ Chile } & Universidades & Técnico & $2-3$ \\
\hline & Centros de formación técnica & Técnico Superior & $2-4$ \\
\hline & Institutos profesionales & Técnico Superior & 2,5 \\
\hline & Institutos técnico-profesionales & Profesional Técnico Superior & \\
\hline \multirow[t]{3}{*}{ Paraguay } & Institutos de educación superiores & Técnico Superior & 2 \\
\hline & Institutos técnicos superiores & Técnico Avanzado & 2 \\
\hline & & Técnico Profesional & 2 \\
\hline \multirow[t]{5}{*}{ Uruguay } & Institutos de Ed. Superior (ITS) & Tecnicaturas/Carreras Técnicas & 2 \\
\hline & Escuelas Técnicas & Tecnólogo & 3 \\
\hline & Escuelas Agrarias & Tecnicaturas/ingenierías/ Licenciaturas & $2-4$ \\
\hline & Universidades & Licenciaturas/ Ingenierías & 4 \\
\hline & Institutos Técnicos Regionales (ITR) & & \\
\hline
\end{tabular}

Fuente: elaboración propia (2020) 
Tabla 2.

Tipo y cantidad de instituciones por gestión (CINE 5)

\begin{tabular}{|c|c|c|c|c|c|}
\hline \multirow{2}{*}{ País } & \multirow{2}{*}{ Tipo de instituciones } & \multicolumn{2}{|l|}{ Estatal } & \multicolumn{2}{|l|}{ Privada } \\
\hline & & Superior & CINE 5 & Superior & CINE 5 \\
\hline \multirow[t]{2}{*}{ Argentina } & Universidades e institutos & 66 & 61 & 65 & 48 \\
\hline & Institutos de educación superior & 1046 & 550 & 1193 & 265 \\
\hline \multirow[t]{4}{*}{ Brasil } & Red Federal de Educación & 639 & & & \\
\hline & Redes Estaduales de Educación & 3003 & $\begin{array}{l}\text { No } \\
\text { corresponde }\end{array}$ & 3.016 & $\begin{array}{l}\text { No } \\
\text { corresponde }\end{array}$ \\
\hline & Redes Municipales de Educación & 268 & & & \\
\hline & Instituciones Privadas & & & & \\
\hline \multirow[t]{2}{*}{ Chile } & Universidad & 25 & 14 & 35 & 12 \\
\hline & $\begin{array}{l}\text { Institutos profesionales, Centros } \\
\text { de formación técnica y FFAA }\end{array}$ & 2 & 2 & 97 & 66 \\
\hline \multirow[t]{2}{*}{ Paraguay } & Universidades & 8 & 4 & 44 & 11 \\
\hline & $\begin{array}{l}\text { Institutos profesionales, Institutos } \\
\text { superiores }\end{array}$ & 58 & 10 & 369 & 253 \\
\hline \multirow[t]{2}{*}{ Uruguay } & $\begin{array}{l}\text { Universidades e Institutos } \\
\text { universitarios }\end{array}$ & 3 & 2 & 16 & 8 \\
\hline & $\begin{array}{l}\text { Institutos de enseñanza terciaria } \\
\text { no universitaria }\end{array}$ & 1 & 1 & 4 & 3 \\
\hline
\end{tabular}

Fuente: elaboración propia (2020)

Considerando el estado de situación descripto, es posible comprender que en los cinco países estudiados, el mapa institucional y de titulaciones resulte heterogéneo. Y esto es así porque en cada uno de ellos existen distintas titulaciones asociadas a la EFTP, con programas que van desde los dos hasta los cinco años de duración, pudiendo estos últimos conducir a grados equiparables a los otorgados en el sector universitario. A esto se suma la dimensión institucional, ya que, a diferencia de la oferta universitaria tradicional, «la educación terciaria técnica se imparte en un conjunto variado de instituciones de naturaleza jurídica distinta, asociada al tipo de titulaciones que están habilitadas a ofrecer» (UNESCO, 2016).

\section{Tendencias emergentes}

La caracterización de la situación a nivel macro de la Educación Superior Tecnológica en los países participantes de Mercosur, permite elaborar algunas comparaciones provisorias y apreciaciones generales que esperan brindar información para avanzar en la definición de políticas para la educación terciaria tecnológica. A continuación se presentan ordenadas en función de las categorías de análisis propuestas:

Oferentes. En todos los países la educación terciaria tecnológica se ofrece en universidades y en instituciones terciarias, tanto de tipo de gestión estatal como privada. Las diferencias entre los países bajo estudio residen en la incidencia de cada tipo de institución. Por ejemplo, la oferta terciaria universitaria tiene un peso menor en Chile y Uruguay en relación con los demás países. Respecto de la oferta universitaria en la región, ocupa un $10 \%$ de la oferta total de la educación terciaria tecnológica, y de ese $10 \%$, dos de cada 
tres instituciones son de gestión privada. Más allá del esquema organizacional que cada país adopte para su provisión, la educación superior o terciaria tecnológica está atravesando un proceso de consolidación como una oferta educativa en un entorno institucional propio y paralelo al universitario que, en muchos casos, se funda en base a ofertas e instituciones de formación profesional que fueron promovidas al nivel terciario.

Duración y título. La duración en todos los casos no es menor a dos años, aunque el máximo varía de tres a cuatro (la carrera universitaria en Chile es la de mayor extensión). Se destaca el caso de Brasil, es posible que los cursos (técnicos subsequentes) tengan duración entre 1 y 2 años, y tienen carga-horaria definida en el «Catálogo Nacional de Cursos Técnicos». Respecto de los títulos obtenidos, existe heterogeneidad de denominaciones entre los cinco países: para las carreras en instituciones no universitarias se obtiene el título de «técnico», «técnico avanzado» o «tecnólogo». Respecto de las carreras universitarias, puede otorgarse el título de «licenciado» $\mathrm{y}$ «técnico de nivel superior o técnico universitario» (caso de Chile).

Cobertura. Los datos de matrícula específica del segmento que corresponde a CINE 5 para la educación terciaria tecnológica no se encuentran disponibles, no obstante, se pueden analizar otros indicadores que brindan algunas coordenadas con relación a la cobertura de estos países y sus diferencias. En el porcentaje de estudiantes en la educación superior de nivel CINE 5 se observa considerables diferencias entre los países analizados. Con mayor cobertura se encuentran Argentina y Chile con el 29\%; le sigue con menos de la mitad de este porcentaje Uruguay con el 13,7 \%, y con marcada diferencia, dando cuenta de la escasa matricula existente para este segmento se encuentran Paraguay y Brasil con el 5,4 \%, y el o,04 \% respectivamente. El porcentaje de jóvenes entre 18 y 24 años que están escolarizados también varía considerablemente: Chile, con la relación más alta, muestra un 52,1 \% de jóvenes escolarizados; le sigue Argentina con el 46,5 \%; Uruguay con el 40,3 \%; Paraguay con el 39,5 y, por último, Brasil con el 30,7 $\%$. Por otra parte, considerando los datos de la Red Iberoamericana de Indicadores de Educación Superior (Red IndicES) y la EPH 2016 en Paraguay, se observa una tasa bruta de matrícula en la educación superior que es superior en Chile y Argentina (63,2 \% y 61,4 $\%$ respectivamente, seguidas por Uruguay con el 50,5 \%; Brasil con el 37,2 \% y Paraguay $34,1 \%$. Las ofertas de educación terciaria tecnológica representan una porción minoritaria dentro de la educación superior.

Organización y enfoque curricular. Se observan distintos niveles de regulación del curriculum, diferente espacio explícito para prácticas y una heterogénea presencia de pasantías profesionales como parte de la formación. Chile, Paraguay y Uruguay explicitan el enfoque por competencias. Los procesos de ingreso son variados e incluyen admisión selectiva, número máximo de vacantes, exámenes y otros mecanismos, así como el cobro de aranceles en el sector privado. La organización de la enseñanza abarca desde propuestas de tiempo completo o tiempo parcial, de la formación presencial a la formación a distancia.

Estructura y Gobierno. Respecto de la estructura del sistema en cada país, la oferta de EFTP formal puede ser parte impartida en el nivel secundario o medio, postsecundario no terciario y/o superior o terciario. Prevalece un modelo de organización a nivel gobierno compuesto por subsistemas cuyas metas son similares, pero las acciones que desarrollan y dependen de un mismo organismo, estás desconectadas, es el caso de Argentina, 
Paraguay y de Brasil. Un aspecto importante para considerar es la regionalización de la oferta universitaria en Chile, con el despliegue de sedes en las distintas ciudades.

Legislación. Se destaca el caso de Argentina que, además de contar con el marco general de una Ley Nacional de Educación al igual de los demás países, se ha sancionado legislación específica: la Ley de Educación Técnica Profesional $\mathrm{N}^{\circ}$ 26.058 que alcanza a todas las instituciones, de todos los niveles, que imparten esta modalidad educativa. En cambio, en Uruguay, Paraguay, Chile y Brasil la normativa específica sobre el desarrollo de la educación terciaria tecnológica se corresponde a los diferentes subsistemas que participan en la oferta.

Financiamiento. En cuatro de los cinco países relevados existe oferta estatal de educación terciaria tecnológica garantizada por presupuesto nacional y gratuito para los estudiantes. En el caso de Chile existe un sistema de becas y gratuidad para los estudiantes de menores ingresos, si cumplen con requisitos académicos.

Sistemas de información. Si bien en algunos países, como Argentina, Brasil y Chile, se detectaron avances en términos de producción y publicación de información, los restantes países presentan algunas limitaciones. Se evidencia la escasa disponibilidad de estadísticas y diagnósticos nacionales relacionada con la heterogeneidad institucional de la oferta y la desarticulación de las instancias de gobierno y producción de la información. Un estudio realizado en la región sobre los sistemas de información de la ETP permite ampliar las dificultades señaladas para el acceso a información y a indicadores básicos (Mejer, 2013, citado en Briasco y otres, 2020).

Mercosur: En todos los países se reconocen avances en acuerdos para la acreditación de saberes por competencias y principalmente por la acreditación y certificación de carreras amparados a los acuerdos regionales y se visualiza como tema prioritario la profundización en estas discusiones acerca de equivalencias, reconocimientos de títulos y revalidas con miras al ejercicio profesional en los distintos países del Mercosur. No obstante, un tema prioritario es el avance en modalidades de trabajo interinstitucional, así como construir viabilidad en un modelo de trabajo interinstitucional y supranacional dada la importancia, necesidad y utilidad del trabajo conjunto de los países del bloque en materia de Educación Tecnológica.

\section{Aportes para la discusión}

Uno de los temas clave que surge es el papel de los sistemas de información para la construcción de políticas, nacionales con perspectiva subregional. De manera coincidente, los cinco países analizados, con situaciones y requerimientos específicos, demandan políticas públicas que revisen las estructuras de gobierno a cargo de la oferta para conectar y articular sus áreas, que garantice coherencia y fortalezca la educación terciaria tecnológica. Se trata de promover y facilitar la coordinación entre las áreas de gobierno involucradas y fortalecer sus capacidades técnicas y administrativas para la gestión y la financiación de estas ofertas. En esa línea, sería necesario avanzar en una normativa que desarrolle en cada país los principales lineamientos político-pedagógicos de la educación terciaria tecnológica a través de una regulación nacional y específica de la modalidad, en perspectiva supranacional. 
Asimismo, para efectivizar procesos tanto de planificación como de evaluación y mejora permanente de la oferta educación terciaria tecnológica se hace indispensable elaborar «Sistemas de Información (SINFO) de la Educación y Formación Técnica y Profesional y del total de su oferta» que brinden estadísticas y diagnósticos nacionales. Tal como se ha afirmado en el artículo, si bien existen operativos censales de los sistemas educativos en general, los datos de la educación técnica del nivel secundario y superior técnico relevados no cuentan con la desagregación requerida para su identificación y, en algunos casos, no se integran con la información recogida por el sector o subsistema. Es necesario contar con información acerca de las instituciones oferentes y los principales indicadores educativos para la toma de decisiones, que estén orientadas al aseguramiento de la calidad y la acreditación, así como al despliegue de acciones para ampliar la equidad en el acceso a la educación terciaria tecnológica.

Otro aporte a la discusión supondría encarar el desafío de formular una mirada integral, que implique la generación de una política en el plano nacional, enmarcada en procesos supranacionales, con miras a formular estrategias y criterios de carácter sistémico que permitan superar la actual fragmentación, la superposición de ofertas en algunos sectores productivos y la ausencia de las mismas en otros. Esto potenciado por la débil articulación e integración entre la educación terciaria y la universitaria permitiendo la construcción de trayectorias formativas. Estas políticas asegurarían la libre circulación de credenciales, situación ya mencionada en las tendencias. Un tema prioritario es la profundización de estrategias para trabajo interinstitucional, así como construir viabilidad para un modelo de gestión supranacional para el bloque Mercosur.

Por último, estudios como el presentado, que den cuenta de desarrollos institucionales, sus resultados e impactos tanto a nivel nacional como regional y supranacional, buscan contribuir a un proceso de mejoramiento en la construcción de políticas públicas.

\section{Referencias}

Afonso, A. y Gonzalez, W. (2018) Desafios da educação profissional e tecnológica: novas faces dos mesmos problemas. ComCiência Revista Eletrônica de Jornalismo Científico, Dossiê emprego e profissões, nov. 2018.

Albornoz, M. y Osorio, L. (2018) Dossier. Información comparable sobre educación superior: el caso del Manual de Lima y la Red INDICES. En Propuesta Educativa: Número 49, Año 27, Vol.1

Briasco, I. (2018) Estudio sobre la educación y formación técnico profesional en Argentina, Brasil y Colombia. Período 2012-2014. Tendencias y situaciones emergentes. Tesis doctoral. Universidad Nacional de Córdoba. Disponible en http://baseries.flacso.org.ar/uploads/productos/1587_01.pdf

Briasco,I. y otres (2020) La oferta de educación terciaria tecnológica. Caracterización de los modelos de gestión institucional desde la perspectiva de los estudios comparados. (Informe Final de Estudios Sectoriales). Inédito. NEIES. SEM. Buenos Aires. Argentina.

Cunha, L. A. (2005) O ensino de ofícios nos primórdios da industrialização. 2. ed. São Paulo: Editora da Unesp. 
Freyre, G. (2014) Casa grande e senzala. São Paulo: Global. Instituto de Estadística de la UNESCO (2011). Clasificación Internacional Normalizada de la Educación CINE 2011. Canada: UIS-UNESCO.

OEI (2017). Manual Iberoamericano de Indicadores de Educación Superior: Manual de Lima. Buenos Aires: Organización de Estados Iberoamericanos para la Educación, la Ciencia y la Cultura. Disponible en: http://www.redindices.org/manual-de-lima

OREALC/UNESCO (2013). Estado de situación de los Sistemas de Información (SINFO) de la Educación y Formación Técnica y Profesional (EFTP) en 12 países de América Latina y el Caribe. Santiago, Chile: OREALC/UNESCO

UNESCO (2015) Recomendación Relativa a la Enseñanza y Formación Técnica y Profesional (EFTP). Paris: UNESCO.

UNESCO (2016) Estrategia para la enseñanza y formación técnica y profesional (EFTP) (2016-2021. Paris: UNESCO.

UNESCO (2017) La Enseñanza y Formación Técnico Profesional en América Latina y el Caribe. Una perspectiva regional hacia 2030. UNESCO. Santiago, Chile.

Valle, J. (2013) Supranational Education: A new field of knowledge to adress educational policies in global world. Revista Española de Educación Comparada, 20(07). http://hdl.handle.net/10486/662795 